\title{
Slacklinen zur Förderung von Gleichgewicht, Aufmerksamkeit und Spaß
}

Slacklinen meint das Balancieren auf einem schmalen Band, welches unterschiedlich lang sein und verschieden stark gespannt werden kann. Dieses Balance-Gerät bleibt auch nach vielen Trainings noch spannend, weil jede Slackline sich etwas anders anfühlt. Mit geeigneten Hilfsmitteln, z.B. der Rookie Rope, lässt es sich soweit vereinfachen, dass jede Person - egal ob jung oder alt - sofort Erfolg hat.

\section{Förderung von Gleichgewicht und Aufmerksamkeit}

Grundsätzlich lässt sich sagen, dass ein vielseitiges Gleichgewichtstraining mit verschiedenen Geräten zu den besten Resultaten führt - hier bietet die Slackline dank der großen Verstellbarkeit bereits sehr vielfältige Möglichkeiten.

Ein Gleichgewichtstraining verbessert die neuromuskuläre Ansteuerung dies bedeutet, dass die Nervenbahnen die Muskelfasern effizienter ansteuern können. Durch diese verbesserte Ansteuerung können mehr Muskelfasern in derselben Zeit aktiviert werden und damit die Explosivkraft (ein Beispiel der Explosivkraft wäre die Sprungkraft) gesteigert werden (Gruber et al. 2007).

Ein Gleichgewichtstraining unterstützt auch bei der Verletzungsprävention. Es stärkt die Bänder und Sehnen und verbessert dadurch die Stabilität der Gelenke. Studien zeigen, dass die Verletzungsanfälligkeit bei Sportlerlnnen durch ein wöchentliches Gleichgewichtstraining um etwa die Hälfte reduziert werden kann (Knobloch et al. 2005).

Slacklinen erfordert eine hohe Konzentration. Ist für einen Moment die Konzentration weg, ist ein Sturz wahrscheinlich. Die Slacklinerlnnen werden auf spielerische Weise dazu animiert, fokussierter ans Werk zu gehen. Studien zeigen, dass sich durch ein mehrwöchiges Slackline-Training die Fähigkeit, sich auf eine Aufgabe zu fokussieren, verbessert (Rodenkirch 2012).

\section{Das Slacklinen}

Beim Slacklinen bleiben Kinder aus eigenem Antrieb und Freude länger an etwas dran. Sie auf der Slackline zu beobachten, gibt Feedback über deren motorische Fähigkeiten: Wie sind ihre koordinativen Fähigkeiten, stehen sie aufrecht, wie ist die Fuß- und Beinbewegung, was trauen sie sich zu?

Ein Gleichgewichtstraining macht dann Spaß, wenn es einen fordert aber nicht überfordert. Die Rookie Rope ist ein geniales Hilfsmittel, um das Slacklinen zu vereinfachen und so für alle zugänglich zu machen. Dafür wird ein Hilfsseil oder Hilfsband 2-2,5 $\mathrm{m}$ über der Slackline installiert. Daran wird mit einer Seilrolle oder einem Rollkarabiner ein weiteres Seil befestigt (Abb. 1).
Dies erlaubt der Person auf der Slackline, sich in der richtigen Balancier-Position zu befinden.

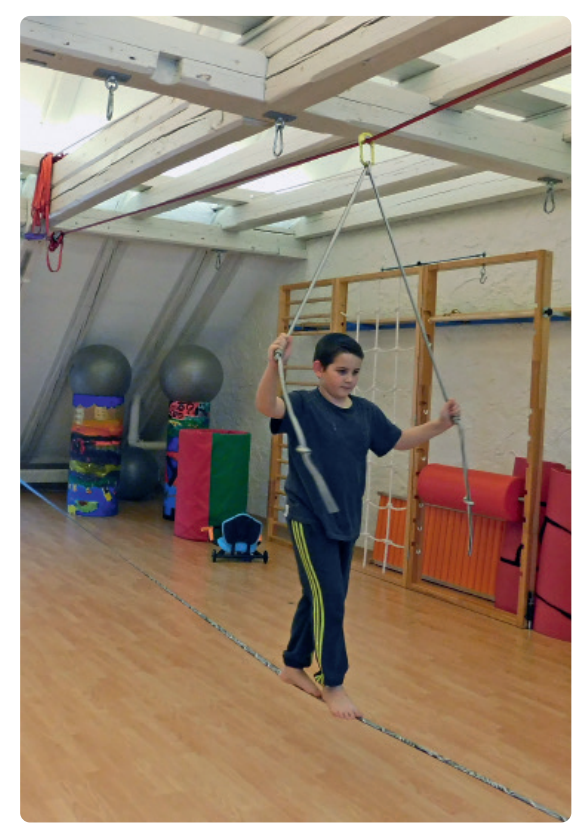

Abb. 1: Rookie Rope

Die Schwierigkeit der Rookie Rope kann auch problemlos angepasst werden:

- Je tiefer man sich am Seil festhält, desto schwieriger wird das Balancieren.

- Wenn das Halteseil mit einer weiteren Seilrolle installiert wird, gleitet es leichter hin und her, was die Challenge erhöht.

- Durch Verdrehen des Halteseils erhöht sich die Reibung, wodurch das Balancieren einfacher wird.

Die Rookie Rope eignet sich sehr gut als Startelement. So kann das Kind sich an das Balancieren auf der Slackline her- 
antasten. Das Halten an den Seilen gibt inm Sicherheit. Das Kind kann dann in einem nächsten Schritt selbst wählen, wann es ohne Halteseile üben möchte. Da der Hangover (Rollkarabiner) gut mit rollt, kann die Bewegung besser erlernt werden, als mit anderen Hilfen von außen.

Spielideen aus den Therapiestunden:

- Blind gehen,

- Schritte zählen,

- Führen - Folgen: Musikalische Begleitung mit drei unterschiedlich klingenden Instrumenten, z. B. Klavier = vorwärts, Trommel $=$ rückwärts, Klang$\mathrm{stab}=$ blind balancieren .

Eine weitere Idee mit der Rookie Rope stellt die Seilbahn dar (Abb. 2). Eine Seilbahn sollte nur benutzt werden, wenn die Installation von Profis eingerichtet wurde. Die Seilbahn eröffnet dem Kind fantasievolle Möglichkeiten, sich auch in der Höhe fortzubewegen und dort z. B. auch eine »Berghütte« zu bauen.

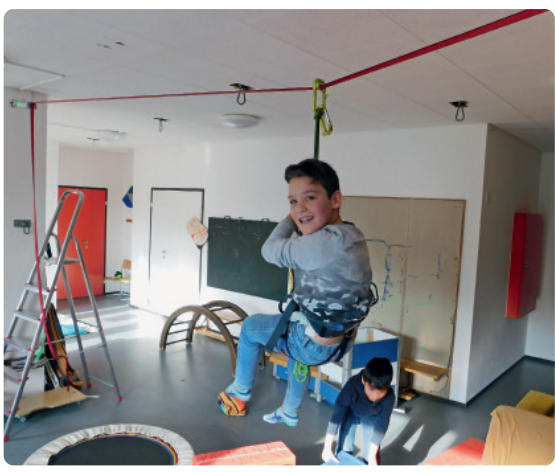

Abb. 2: Seilbahn

\section{Die Grundposition}

Verschiedenste Positionen sind auf der Slackline möglich: vom Balancieren im Sitzen über das Liegen bis hin zum Handstand. Die meisten sind zu Beginn am stärksten davon fasziniert, das Gleichgewicht auf den Füßen zu finden.

Die ersten Versuche sollten auf einer kurzen (4-10 $\mathrm{m}$ lang) und straff gespannten Slackline stattfinden. Der Fuß wird gerade aufs Band gestellt, das zweite Bein seitlich zum Ausbalancieren benutzt. Das Standbein ist angewinkelt. Die Hüfte ist aufrecht, den Blick nach vorne gerichtet. Die Daumen zeigen nach oben, die Schultern sind möglichst entspannt.

Zu Beginn wird das Standbein hin und her zittern; am meisten bei schweren und kräftigen Personen. Bei Kindern tritt dieser Reflex weniger stark auf. Das Zittern ist durch einen monosynaptischen Gleichgewichtsreflex verursacht. Positionssensoren teilen dem Rückenmark mit, dass das Bein etwas zu stark links ist, dadurch wird ein Reflex eingeleitet, der das Bein wieder nach rechts zieht. Dieser Reflex ist meist etwas zu stark, wodurch er dann wieder auf die andere Seite zurück korrigiert werden muss. Durch diesen Reflex werden ganz unbewusst starke Fortschritte im Gleichgewicht erzielt. Die Körperposition wird stabiler und ein erstes Erfolgserlebnis stellt sich ein.

Fortschritte auf der Slackline werden vor allem erzielt, wenn viel Zeit auf dem Band verbracht wird. Wie weit man darauf läuft, ist hingegen weniger relevant. Kindern macht es trotzdem mehr Spaß, die komplette Line durchzulaufen, statt geduldig an Ort und Stelle stehen zu bleiben. Sobald die ersten Schritte gut funktionieren, kann die Schwierigkeit durch das Verändern der Spannung in der Slackline angepasst werden.

\section{Gruppenangebot}

Auf der Slackline lässt sich problemlos auch in Gruppen arbeiten. Die Slackline lässt sich beispielsweise mit einem Kasten unterteilen - dadurch werden aus einer Slackline zwei und es können zwei Kinder gleichzeitig auf einer Slackline sein (Spielidee "Spiegeln«: Beide Kinder nutzen die Halte- seile. Ein Kind gibt die Bewegung vor, das andere spiegelt die Bewegung) Darüber hinaus können sich die Kinder auch gegenseitig helfen: das eine Kind balancierend, das andere Kind als Helfer.

Die Slackline ist ein ideales Sportgerät, um in ein Miteinander zu kommen. Beim Start auf der Slackline ist eine helfende Hand für die Sicherheit von großem Vorteil. Die Verbindung durch die Hand schafft Nähe und Berührung und ermöglicht, den Körper zu spüren und zu merken, was der andere braucht.

\section{Training für Jung und Alt}

Das Slacklinen lässt sich in jedem Alter erlernen. Jeder, der zu Fuß noch gut unterwegs ist, wird mit etwas Übung auch auf der Slackline erfolgreich sein. Dies ist nicht nur in der Therapie-Praxis, sondern vor allem auch in der Natur zwischen Bäumen möglich (Abb. 3). Slacklinen ist ein Sport, der oft positive Emotionen weckt, wie Freude, Lust und Genuss, auch weil man einfach und schnell Bewegung und das Zusammensein an einem schönen Nachmittag im Park erleben kann. Zudem trainiert das Slacklinen die Koordination und Konzentration und wird heutzutage auch regelmäßig in der Physiotherapie - beispielsweise für die Sturzprävention oder Rehabilitation nach einem Kreuzbandriss - eingesetzt.

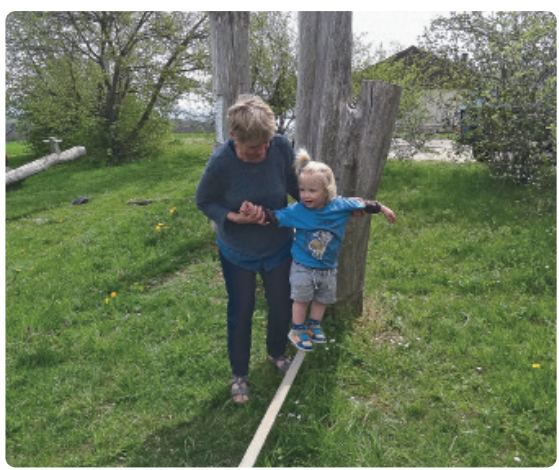

Abb. 3: Draußen Slacklinen bereitet Kindern und Erwachsenen Freude 


\section{Das Material}

Wir empfehlen, eine Slackline mit einer Breite von $25-40 \mathrm{~mm}$ zu verwenden. Breitere Slacklines sind nicht etwa stabiler, sondern kippen nur mehr zur Seite. Slacklinerlnnen können die Kanten nicht mehr ertasten und wissen dadurch nicht genau, wie der Fuß ausgerichtet ist. Als Spannsystem ist eine Ratsche zu empfehlen - es sollte darauf geachtet werden, dass sich diese nicht mitten im System sondern gleich beim Ankerpunkt befindet.

Meist werden Slacklines zwischen Bäumen gespannt. In anderen Räumlichkeiten muss aber eine andere Lösung her. Leider ist nicht jede Struktur geeignet, um Slacklines zu befestigen. Es wirken nämlich Zugkräfte von bis zu $7 \mathrm{kN}(=700 \mathrm{~kg})$. Deshalb sollte die Slackline weder an Sprossenwänden noch an der Heizung montiert werden. Auch Aluminium-Karabiner haben in Slacklines nichts verloren. Strukturen, die sich zur Befestigung eignen, sind:
- Wandhalterungen, welche speziell für Slacklines entwickelt wurden und an Betonwänden befestigt werden sollten, und

- Betonsäulen.

Falls keine Beton-Strukturen vorhanden sind, muss ein Statiker klären, ob und wie eine Slackline befestigt werden kann.

Ein freistehendes Slackline-Gestell kann zwar in jedem Fall eingesetzt werden, ist aber nur begrenzt zu empfehlen, da sich unter der Slackline ein Balken befindet. Außerdem kann keine Rookie Rope darüber gespannt werden kann.

\section{(D) Literatur}

Gruber, M., Gruber, S.B., Taube, W., Schubert, M., Beck, S.C., Gollhofer, A. (2007): Differential effects of ballistic versus sensorimotor training on rate of force development and neural activation in humans. The Jour- nal of Strength \& Conditioning Research 21 (1), 274-282, https://doi. org/10.1519/00124278-20070200000049

Knobloch, K., Martin-Schmitt, S., Gösling, T., Jagodzinski, M. (2005): Prospektives Propriozeptions- und Koordinationstraining zur Verletzungsreduktion im professionellen Frauenfußballsport. Sportverletzung Sportschaden 19 (3), 123-129, https://doi. org $/ 10.1055 / \mathrm{s}-2005-858345$

Rodenkirch, T. (2012): Verbesserung de Konzentrationsfähigkeit mittels Slacklinetraining. Masterarbeit Zürich. In: https://www.wupp24.de/uploads/ tx_bdeventregistrationmgr/master arbeit-tobias-rodenkirch_web.pdf, 24.09.2019

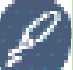
Kontakt Samuel Volery info@slacktivity.ch Anja Mengotti-Hübscher a.mengotti@pmtwl.ch

DOI 10.2378/ mot2020.art26d

\section{Rubriken in der Zeitschrift »motorik» - kurz erklärt}

Für die Rubrik »Forum Psychomotorik« können Praxisbeiträge, Diskussionsbeiträge (gerne in Pro/ContraForm) und Beiträge zu Organisation, Recht und Finanzen eingereicht werden.

Die Rubrik »Fachbeiträge aus Theorie und Praxis« enthält Beiträge aus der Grundlagenforschung, (angewandte) Wissenschaftsbeiträge und Berichte aus evaluierter Praxis. Die
Beiträge dieser Rubrik werden von mindestens zwei FachkollegInnen anonym begutachtet (Peer Review).

Für die Rubrik »Auf den Punkt gebracht « sind Kurzartikel zu aktuellen Themen, Fachbegriffen und Hintergrundwissen willkommen.

Im Service-Teil finden sich unter „Praxistipps« konkrete Anleitungen für die Praxis, unter »Medien und Materialien« u. a. Rezensionen und Kurz- vorstellungen von Büchern, Filmen, Software und Linktipps, unter »Aktuelles / Kurz berichtet« Studien und Qualifikationsarbeiten, Projekt- und Tagungsberichte, Termine etc.

Wir würden Sie gerne als Autorin oder Autor für die »motorik» gewinnen! Nehmen Sie Kontakt mit uns auf: motorik-schriftleitung@reinhardtjournals.de 